\title{
The Heat Kernel on Hyperbolic Space
}

\author{
Alexander Grigor'yan* \\ Department of Mathematics \\ Imperial College, London SW7 2BZ \\ United Kingdom \\ email: a.grigoryan@ic.ac.uk \\ Masakazu Noguchi \\ Department of Mathematics \\ Imperial College, London SW7 2BZ \\ United Kingdom \\ email: m.noguchi@ic.ac.uk
}

September 1997

\section{Introduction and the main result}

The purpose of this note is to provide a new proof for the explicit formulas of the heat kernel on hyperbolic space. By definition, the hyperbolic space $\mathbb{H}^{n}$ is a (unique) simply connected complete $n$-dimensional Riemannian manifold with a constant negative sectional curvature -1 .

Let $\Delta$ denote the Laplacian on a Riemannian manifold $X$. The heat kernel on $X$ is a function $p(x, y, t)$ on $X \times X \times(0, \infty)$ which is the minimal positive fundamental solution to the heat equation

$$
\frac{\partial v}{\partial t}=\Delta v
$$

In other words, the Cauchy problem

$$
\left\{\begin{array}{l}
\frac{\partial v}{\partial t}=\Delta v \\
\left.v\right|_{t=0}=v_{0}(x)
\end{array}\right.
$$

has a solution

$$
v(x, t)=\int_{X} p(x, y, t) v_{0}(y) d y
$$

*Supported by EPSRC Fellowship B/94/AF/1782 
provided that $v_{0}$ is a bounded continuous function. If, in addition, $v_{0} \geq 0$, then (1.2) defines the minimal positive solution to (1.1) (see [4] for details).

If $X$ is the Euclidean space $\mathbb{R}^{n}$ then the heat kernel is given by the classical formula

$$
p(x, y, t)=\frac{1}{(4 \pi t)^{n / 2}} \exp \left(-\frac{\rho^{2}}{4 t}\right),
$$

where $\rho=|x-y|$. Due to homogeneity of the hyperbolic space, the heat kernel on $\mathbb{H}^{n}$ also depends only on $t$ and $\rho$ (where $\rho=\operatorname{dist}(x, y)$ is now the geodesic distance on $\left.\mathbb{H}^{n}\right)$. Let us denote the heat kernel on $\mathbb{H}^{n}$ by $p_{n}(\rho, t)$. Then we have the following.

Theorem 1.1 The heat kernel $p_{n}(\rho, t)$ on the hyperbolic space $\mathbb{H}^{n}$ is given by the following formulas.

$$
\begin{aligned}
& \text { If } n=2 m+1 \text {, then } \\
& \qquad p_{n}(\rho, t)=\frac{(-1)^{m}}{2^{m} \pi^{m}} \frac{1}{(4 \pi t)^{\frac{1}{2}}}\left(\frac{1}{\sinh \rho} \frac{\partial}{\partial \rho}\right)^{m} e^{-m^{2} t-\frac{\rho^{2}}{4 t}} . \\
& \text { If } n=2 m+2 \text {, then } \\
& p_{n}(\rho, t)=\frac{(-1)^{m}}{2^{m+\frac{5}{2}} \pi^{m+\frac{3}{2}}} t^{-\frac{3}{2}} e^{-\frac{(2 m+1)^{2}}{4} t}\left(\frac{1}{\sinh \rho} \frac{\partial}{\partial \rho}\right)^{m} \int_{\rho}^{\infty} \frac{s e^{-\frac{s^{2}}{4 t}}}{(\cosh s-\cosh \rho)^{\frac{1}{2}}} d s .
\end{aligned}
$$

In particular, if $n=1$, then (1.4) coincides with the one-dimensional Euclidean heat kernel (1.3). If $n=3$, then (1.4) becomes

$$
p_{3}(\rho, t)=\frac{1}{(4 \pi t)^{\frac{3}{2}}} \frac{\rho}{\sinh \rho} e^{-t-\frac{\rho^{2}}{4 t}}
$$

whereas (1.5) yields, for $n=2$,

$$
p_{2}(\rho, t)=\frac{\sqrt{2}}{(4 \pi t)^{\frac{3}{2}}} e^{-\frac{1}{4} t} \int_{\rho}^{\infty} \frac{s e^{-\frac{s^{2}}{4 t}}}{(\cosh s-\cosh \rho)^{\frac{1}{2}}} d s .
$$

The formulas (1.4) and (1.5) are not new. The heat kernel in dimension two (formula (1.7)) was found by McKean [6] (see also [1, pp. 242-246]). The three-dimensional case (formula (1.6)) was proved in [3, p. 396]. For $n>3$, the formulas (1.4) and (1.5) can be obtained inductively by using the recurrence relation

$$
p_{n+2}(\rho, t)=-\frac{\exp (-n t)}{2 \pi \sinh \rho} \frac{\partial}{\partial \rho} p_{n}(\rho, t) .
$$

The identity (1.8) is attributed in [3, p. 396] to Millson (unpublished). Its proof can be found in [2, Theorem 2.1].

In this note, we provide an independent proof of (1.4) and (1.5) by using a completely different approach, based on the following two ingredients: 
(1) the relation between the heat kernel and the wave kernel which follows from the spectral theory;

(2) the explicit formula for the wave kernel on symmetric spaces which is found in $[5]$.

These are enough to derive (1.4) and (1.5) directly, without using (1.8).

\section{Proof of the main theorem}

Crucial for the proof is the following relation between the heat equation and the wave equation. Let $L$ denote an elliptic operator on a manifold $X$, and let us consider the Cauchy problem for the wave equation in $X \times(-\infty, \infty)$,

$$
\left\{\begin{array}{l}
\frac{\partial^{2} u}{\partial t^{2}}=L u \\
\left.u\right|_{t=0}=u_{0}(x) \\
\left.\frac{\partial u}{\partial t}\right|_{t=0}=0
\end{array}\right.
$$

for a given $u_{0} \in C(X)$. Assuming that the operator $L$ is initially defined on $C_{0}^{\infty}(X)$ and has a self-adjoint non-positive definite extension in $\mathcal{L}^{2}(X)$ (which will also be denoted by $L)$, the solution to (2.1) can be represented as

$$
u(x, t)=\cos (t \sqrt{-L}) u_{0}(x) .
$$

Similarly, for the Cauchy problem for the heat equation in $X \times(0, \infty)$

$$
\left\{\begin{array}{l}
\frac{\partial v}{\partial t}=L v \\
\left.v\right|_{t=0}=v_{0}(x)
\end{array}\right.
$$

one has

$$
v(x, t)=\exp (t L) v_{o}(x), \quad t>0 .
$$

On the other hand, we have the following Fourier transform identity (where $t>0)$ :

$$
e^{-t \lambda^{2}}=\frac{1}{(4 \pi t)^{\frac{1}{2}}} \int_{-\infty}^{\infty} e^{-\frac{s^{2}}{4 t}} e^{i s \lambda} d s=\frac{1}{(4 \pi t)^{\frac{1}{2}}} \int_{-\infty}^{\infty} e^{-\frac{s^{2}}{4 t}} \cos (s \lambda) d s,
$$

where $\lambda$ is either a real number or a self-adjoint operator. Put $\lambda=\sqrt{-L}$; then

$$
e^{t L}=\frac{1}{(4 \pi t)^{\frac{1}{2}}} \int_{-\infty}^{\infty} e^{-\frac{s^{2}}{4 t}} \cos (s \sqrt{-L}) d s
$$

whence we have the following proposition.

Proposition 2.1 Let $L$ be as above, and let $v_{0}=u_{0}$ be a bounded continuous function on $X$. Then the solutions $u(x, t)$ of (2.1) and $v(x, t)$ of (2.2) are related as follows:

$$
v(x, t)=\frac{1}{(4 \pi t)^{\frac{1}{2}}} \int_{-\infty}^{\infty} e^{-\frac{s^{2}}{4 t}} u(x, s) d s .
$$


Let us describe the next ingredient of the proof: the explicit formula for $u(x, s)$ on $\mathbb{H}^{n}$. Let us denote by $S_{r}(x)$ the geodesic sphere on $\mathbb{H}^{n}$ with centre $x \in \mathbb{H}^{n}$ and radius $r$. It is known that the area of $S_{r}(x)$ is equal to $A(r)=$ $\Omega_{n} \sinh ^{n-1} r$, where

$$
\Omega_{n}:=\frac{2 \pi^{\frac{n}{2}}}{\Gamma\left(\frac{n}{2}\right)}
$$

is the area of the unit sphere in $\mathbb{R}^{n}$. For any number $r>0$, denote by $M^{r}$ the averaging operator on $\mathbb{H}^{n}$ :

$$
\left(M^{r} f\right)(x):=\frac{1}{A(r)} \int_{S_{r}(x)} f(y) d \omega(y),
$$

where $d \omega$ is the area element of $S_{r}(x)$. For convenience, let us denote

$$
\left(N_{m, k}^{r} f\right)(x):=\left(\frac{\partial}{\partial \cosh r}\right)^{m}\left(M^{r} f(x) \sinh ^{k} s\right),
$$

where $k$ and $m$ are non-negative integers.

The next assertion follows from the general mean value theorem for symmetric spaces and can be found in [5, Chapter 2].

Proposition 2.2 Let $u(x, s)$ be the solution of the Cauchy problem on $\mathbb{H}^{n} \times$ $(-\infty, \infty)$

$$
\left\{\begin{array}{l}
L u=0 \\
\left.u\right|_{t=0}=u_{0}, \\
\left.\frac{\partial u}{\partial t}\right|_{t=0}=u_{1},
\end{array}\right.
$$

where

$$
L=\Delta+\left(\frac{n-1}{2}\right)^{2}
$$

and $u_{0}$ and $u_{1}$ are continuous initial functions. Then for any $x \in \mathbb{H}^{n}$ and $s>0$, we have the following.

If $n \geq 3$ is odd, then

$$
u(x, s)=c_{n}\left(\frac{\partial}{\partial s} N_{\frac{n-3}{2}, n-2}^{s} u_{0}+N_{\frac{n-3}{2}, n-2}^{s} u_{1}\right)
$$

where

$$
c_{n}=\frac{\Omega_{n}}{2(n-3) ! ! \Omega_{n-1}}=\frac{1}{(n-2) ! !} .
$$

(We use the notation $k ! !:=1 \cdot 3 \cdot 5 \cdot \ldots \cdot k$ if $k$ is odd, $k ! !=2 \cdot 4 \cdot 6 \cdot \ldots \cdot k$ if $k$ is even and $k ! !=1$ if $k \leq 0$.)

If $n \geq 2$ is even, then the following equation holds:

$$
\frac{1}{2} \int_{0}^{s} \frac{u(x, r)+u(x,-r)}{(\cosh s-\cosh r)^{\frac{1}{2}}} d r=c_{n} N_{\frac{n-2}{2}, n-2}^{s} u_{0}
$$

where $c_{n}=\frac{\Omega_{n}}{\sqrt{2}(n-3) ! ! \Omega_{n-1}}=\frac{\pi}{2(n-2) ! !}$. 
Proposition 2.2 simplifies for the case $u_{1}=0$, as follows.

Corollary 2.3 Assume, under the hypotheses of Proposition 2.2, that $u_{1}=0$. Then, for any $x \in \mathbb{H}^{n}$ and $s>0$, we have the following.

If $n \geq 3$ is odd, then

$$
u(x, s)=c_{n} \frac{\partial}{\partial s} N_{\frac{n-3}{2}, n-2}^{s} u_{0}(x) .
$$

If $n \geq 2$ is even, then

$$
u(x, s)=\frac{c_{n}}{\pi} \frac{\partial}{\partial s} \int_{0}^{s} \frac{\sinh \rho}{(\cosh s-\cosh \rho)^{\frac{1}{2}}} N_{\frac{n-2}{2}, n-2}^{\rho} u_{0}(x) d \rho
$$

Proof. Since (2.11) is obvious from (2.9), let us concentrate on (2.12). Observe that $u_{1}=0$ implies that the solution $u(x, t)$ is an even function in $t$. Therefore (2.10) acquires the form

$$
\int_{0}^{s} K(s, r) u(x, r) d r=c_{n} N_{\frac{n-2}{2}, n-2}^{s} u_{0}(x),
$$

where we denote

$$
K(s, r):=\frac{1}{(\cosh s-\cosh r)^{\frac{1}{2}}}, \quad s \neq r .
$$

By multiplying (2.13) by $K(t, s) \sinh s$ and integrating in $s$, we have, for any $t$ $>0$,

$\int_{0}^{t} K(t, s) \sinh s \int_{0}^{s} u(x, r) K(s, r) d r d s=c_{n} \int_{0}^{t} K(t, s) \sinh s N_{\frac{n-2}{2}, n-2}^{s} u_{0}(x) d s$.

The left-hand side is computed by changing the order of integration:

$$
\int_{0}^{t} d r \int_{r}^{t} u(x, r) K(t, s) K(s, r) \sinh s d s=\pi \int_{0}^{t} u(x, r) d r
$$

where we have used the identity

$$
\int_{r}^{t} K(t, s) K(s, r) \sinh s d s=\int_{a}^{b} \frac{d \xi}{\sqrt{(b-\xi)(\xi-a)}}=\pi .
$$

By (2.14) and (2.15),

$$
\pi \int_{0}^{t} u(x, r) d r=c_{n} \int_{0}^{t} K(t, s) \sinh s N_{\frac{n-2}{2}, n-2}^{s} u_{0}(x) d s
$$

whence we obtain (2.12) by differentiating in $t$.

Our next goal will be to extend (2.11) and (2.12) to the case $s<0$. Fix $x$, and denote, for any $\rho>0$,

$$
U(\rho):=\int_{S_{\rho}(x)} u_{0}(y) d \omega(y)=A(\rho) M^{\rho} u_{0}(x),
$$

and extend $U(\rho)$ to non-positive $\rho$ by $U(0)=0$ and $U(\rho)=U(|\rho|)$. 
Lemma 2.4 We have $U(r) \in C^{n-1}(-\infty, \infty)$. Moreover, $U^{(l)}(0)=0$ for any $l=0,1, \ldots, n-1$.

Proof. The function $u_{0}$ is infinitely smooth, and so is $U(\rho)$ for $\rho \neq 0$. To handle the case $\rho=0$, let us rewrite (2.16) in the polar coordinates $y=(\rho, \theta)$ centred at $x$ :

$$
U(\rho)=\int_{\mathbb{S}^{n}} u_{0}(\rho, \theta) A(\rho) d \theta
$$

The trouble is that the distance function $\rho(y):=\operatorname{dist}(x, y)$ is not smooth at $y=x$. However, its square $\rho^{2}$ is infinitely smooth in $y$, which implies that the function $u_{0}$ is $C^{\infty}$ in $\rho^{2}$. Since

$$
\frac{\partial}{\partial \rho}=2 \rho \frac{\partial}{\partial\left(\rho^{2}\right)}
$$

it is not difficult to see by induction that $\left(\frac{\partial}{\partial \rho}\right)^{k}$ is a sum of the terms proportional to

$$
\rho^{2 j-k}\left(\frac{\partial}{\partial\left(\rho^{2}\right)}\right)^{j}
$$

where $j=1,2, \ldots, k$.

Obviously, the function $\left(\frac{\partial}{\partial \rho}\right)^{m} A$ has, at $\rho=0$, a zero of order $\rho^{n-1-m}$, that is, it can be represented as

$$
\left(\frac{\partial}{\partial \rho}\right)^{m} A=\rho^{n-1-m} A_{m}(\rho)
$$

where $A_{m}(\rho)$ is a continuous function of $\rho$. When differentiating $l$ times the product $u_{0}(\rho, \theta) A(\rho)$ in $\rho$, we have the sum of the terms proportional to

$$
\left(\frac{\partial}{\partial \rho}\right)^{k} u_{0} \cdot\left(\frac{\partial}{\partial \rho}\right)^{l-k} A
$$

which splits further, to the sum of terms as

$$
\rho^{2 j-k}\left(\frac{\partial}{\partial\left(\rho^{2}\right)}\right)^{j} u_{0} \cdot \rho^{n-1-l+k} A_{l-k}(\rho)=\rho^{2 j+(n-1-l)}\left(\frac{\partial}{\partial\left(\rho^{2}\right)}\right)^{j} u_{0} \cdot A_{l-k}(\rho) .
$$

If $l \leq n-1$, then the latter function is continuous up to $\rho=0$ and vanishes at $\rho=0$ whence we obtain $U \in C^{n-1}$ and $U(0)=U^{\prime}(0)=U^{\prime \prime}(0)=\ldots=$ $U^{(n-1)}(0)=0$.

Corollary 2.5 We have the following, under the hypotheses of Corollary 2.3, for all $x \in \mathbb{H}^{n}$ and $s \in(-\infty, \infty)$.

If $n \geq 3$ is odd, then

$$
u(x, s)=\frac{c_{n}}{\Omega_{n}} \frac{\partial}{\partial s}\left(\frac{1}{\sinh s} \frac{\partial}{\partial s}\right)^{\frac{n-3}{2}}\left[\frac{1}{\sinh s} U(s)\right] .
$$




$$
\text { If } n \geq 2 \text { is even, then }
$$

$$
u(x, s)=\frac{c_{n}}{\pi \Omega_{n}} \frac{\partial}{\partial s} \int_{0}^{s} K(s, \rho) \sinh \rho\left(\frac{1}{\sinh \rho} \frac{\partial}{\partial \rho}\right)^{\frac{n-2}{2}}\left(\frac{1}{\sinh \rho} U(\rho)\right) d \rho .
$$

Proof. Indeed, (2.17) coincides with (2.11) if $s>0$. Since both sides of (2.17) are even functions in $s,(2.17)$ holds also for $s<0$. The case $s=0$ follows by continuity. Each derivation $\frac{\partial}{\partial s}$ of $U(s)$, or division by $\sinh s$, reduces smoothness at 0 by at most 1 . After $n-1$ such operations as in (2.17), we still have a continuous function on the right-hand side of (2.17). In particular, this argument shows that the right-hand side of (2.17) has meaning for $s=0$.

The equation (2.18) follows in the same way from (2.12).

Proof of Theorem 1.1. It is clear from (2.8) that

$$
e^{t \Delta}=\exp \left(-\left(\frac{n-1}{2}\right)^{2} t\right) e^{t L}
$$

so the kernels of the semigroups $e^{t \Delta}$ and $e^{t L}$ are related by the same equation. Therefore it will suffice to find the kernel of the semigroup $e^{t L}$. Note that the operator $L$ is non-positive definite, for the top of the spectrum of $\Delta$ in $\mathcal{L}^{2}\left(\mathbb{H}^{n}\right)$ is equal to $-\left(\frac{n-1}{2}\right)^{2}$.

We shall use the notation $u(x, t)$ and $v(x, t)$ for the solutions to the initial problems (2.1) and (2.2), respectively, for the operator (2.8). The initial function $u_{0}=v_{0}$ is supposed to be in $C_{0}^{\infty}\left(\mathbb{H}^{n}\right)$.

Case I: $n=2 m+1$.

We have, by (2.3) and (2.17),

$$
v(x, t)=\frac{c_{n}}{(4 \pi t)^{\frac{1}{2}}} \int_{-\infty}^{\infty} e^{-\frac{s^{2}}{4 t}} \frac{\partial}{\partial s}\left(\frac{1}{\sinh s} \frac{\partial}{\partial s}\right)^{\frac{n-3}{2}}\left[\frac{1}{\sinh s} U(s)\right] d s,
$$

whence we obtain, by integration by parts,

$$
\begin{aligned}
v(x, t) & =\frac{c_{n} \Omega_{n}^{-1}}{(4 \pi t)^{\frac{1}{2}}} \int_{-\infty}^{\infty} e^{-\frac{s^{2}}{4 t}} \frac{\partial}{\partial s}\left(\frac{1}{\sinh s} \frac{\partial}{\partial s}\right)^{m-1}\left[\frac{1}{\sinh s} U(s)\right] d s \\
& =\frac{(-1)^{m} c_{n} \Omega_{n}^{-1}}{(4 \pi t)^{\frac{1}{2}}} \int_{-\infty}^{\infty}\left\{\left(\frac{1}{\sinh s} \frac{\partial}{\partial s}\right)^{m} e^{-\frac{s^{2}}{4 t}}\right\} U(s) d s \\
& =\frac{2(-1)^{m} c_{n} \Omega_{n}^{-1}}{(4 \pi t)^{\frac{1}{2}}} \int_{0}^{\infty}\left\{\left(\frac{1}{\sinh s} \frac{\partial}{\partial s}\right)^{m} e^{-\frac{s^{2}}{4 t}}\right\} U(s) d s \\
& =\frac{2(-1)^{m} c_{n} \Omega_{n}^{-1}}{(4 \pi t)^{\frac{1}{2}}} \int_{\mathbb{H}^{n}}\left(\frac{1}{\sinh \rho} \frac{\partial}{\partial \rho}\right)^{m} e^{-\frac{\rho^{2}}{4 t}} u_{0}(y) d y,
\end{aligned}
$$

where $\rho=\operatorname{dist}(x, y)$.

Observing that

$$
2 c_{n} \Omega_{n}^{-1}=\frac{1}{(n-3) ! ! \Omega_{n-1}}=\frac{1}{2^{m} \pi^{m}},
$$


we conclude that the operator $e^{t L}$ is an integral operator with kernel

$$
\frac{(-1)^{m}}{2^{m} \pi^{m}} \frac{1}{(4 \pi t)^{\frac{1}{2}}}\left(\frac{1}{\sinh \rho} \frac{\partial}{\partial \rho}\right)^{m} e^{-\frac{\rho^{2}}{4 t}} .
$$

Therefore the heat kernel $p_{n}(\rho, t)$ of $e^{t \Delta}$ is

$$
p_{n}(\rho, t)=\frac{(-1)^{m}}{2^{m} \pi^{m}} \frac{1}{(4 \pi t)^{\frac{1}{2}}}\left(\frac{1}{\sinh \rho} \frac{\partial}{\partial \rho}\right)^{m} e^{-m^{2} t-\frac{\rho^{2}}{4 t}},
$$

which was to be proved.

Case II: $n=2 m+2$.

We have, by (2.3) and (2.18), by changing the order of the integrals and by integration by parts,

$$
\begin{aligned}
& v(x, t) \\
= & \frac{c_{n}^{\prime}}{\sqrt{t}} \int_{-\infty}^{\infty} e^{-\frac{s^{2}}{4 t}}\left[\frac{\partial}{\partial s} \int_{0}^{s} K(s, \rho) \sinh \rho\left(\frac{1}{\sinh \rho} \frac{\partial}{\partial \rho}\right)^{m}\left(\frac{1}{\sinh \rho} U(\rho)\right) d \rho\right] d s \\
= & \frac{c_{n}^{\prime}}{\sqrt{t}} \int_{-\infty}^{\infty} \frac{2 s}{4 t} e^{-\frac{s^{2}}{4 t}}\left[\int_{0}^{s} K(s, \rho) \sinh \rho\left(\frac{1}{\sinh \rho} \frac{\partial}{\partial \rho}\right)^{m}\left(\frac{1}{\sinh \rho} U(\rho)\right) d \rho\right] d s \\
= & \frac{c_{n}^{\prime}}{t^{\frac{3}{2}}} \int_{0}^{\infty}\left(\int_{\rho}^{\infty} s e^{-\frac{s^{2}}{4 t}} K(s, \rho) d s\right) \sinh \rho\left(\frac{1}{\sinh \rho} \frac{\partial}{\partial \rho}\right)^{m}\left(\frac{1}{\sinh \rho} U(\rho)\right) d \rho \\
= & \frac{(-1)^{m} c_{n}^{\prime}}{t^{\frac{3}{2}}} \int_{0}^{\infty}\left(\frac{1}{\sinh \rho} \frac{\partial}{\partial \rho}\right)^{m}\left(\int_{\rho}^{\infty} s e^{-\frac{s^{2}}{4 t}} K(s, \rho) d s\right) U(\rho) d \rho \\
= & \frac{(-1)^{m} c_{n}^{\prime}}{t^{\frac{3}{2}}} \int_{\mathbb{H}^{n}}\left(\left(\frac{1}{\sinh \rho} \frac{\partial}{\partial \rho}\right)^{m} \int_{\rho}^{\infty} s e^{-\frac{s^{2}}{4 t}} K(s, \rho) d s\right) u_{0}(y) d y,
\end{aligned}
$$

where $c_{n}^{\prime}=\frac{c_{n}}{2 \pi^{\frac{3}{2}} \Omega_{n}}=\frac{1}{2^{m+\frac{5}{2}} \pi^{m+\frac{3}{2}}}$.

This shows that the kernel of the integral operator $e^{t L}$ is

$$
\frac{(-1)^{m}}{2^{m+\frac{5}{2}} \pi^{m+\frac{3}{2}}} t^{-\frac{3}{2}}\left(\frac{1}{\sinh \rho} \frac{\partial}{\partial \rho}\right)^{m} \int_{\rho}^{\infty} \frac{s e^{-\frac{s^{2}}{4 t}}}{(\cosh s-\cosh \rho)^{\frac{1}{2}}} d s
$$

whence the heat kernel is

$p_{2 m+2}(x, y, t)=\frac{(-1)^{m}}{2^{m+\frac{5}{2}} \pi^{m+\frac{3}{2}}} e^{-t \frac{(2 m+1)^{2}}{4}} t^{-\frac{3}{2}}\left(\frac{1}{\sinh \rho} \frac{\partial}{\partial \rho}\right)^{m} \int_{\rho}^{\infty} \frac{s e^{-\frac{s^{2}}{4 t}}}{(\cosh s-\cosh \rho)^{\frac{1}{2}}} d s$.

\section{References}

[1] Chavel I. , "Eigenvalues in Riemannian geometry" Academic Press, New York, 1984.

[2] Davies E.B., Mandouvalos N. , Heat kernel bounds on hyperbolic space and Kleinian groups, Proc. London Math. Soc.(3) , 52 (1988) no.1, 182208. 
[3] Debiard A., Gaveau B., Mazet E. , Théorèmes de comparison in gèomètrie riemannienne, Publ. Kyoto Univ. , 12 (1976) 391-425.

[4] Dodziuk J. , Maximum principle for parabolic inequalities and the heat flow on open manifolds, Indiana Univ. Math. J. , 32 (1983) no.5, 703-716.

[5] Helgason S. , "Groups and geometric analysis" Academic Press, New York, 1984.

[6] McKean H.P. , An upper bound to the spectrum of $\Delta$ on a manifold of negative curvature, J. Diff. Geom., 4 (1970) 359-366. 